\title{
Continuous process of carbon nanotubes synthesis by decomposition of methane using an arc-jet plasma
}

\author{
S. I. Choi*, J. S. Nam, J. I. Kim, T. H. Hwang, J. H. Seo, and S. H. Hong \\ Department of Nuclear Engineering, Seoul National University \\ Seoul 151-742, Korea
}

\begin{abstract}
We present a method of producing carbon nanotubes by means of the thermal plasma decomposition of methane $\left(\mathrm{CH}_{4}\right)$ in an arc-jet plasma of high temperature (5000-20000 K). Carbon nanotubes are produced under a floating condition by introducing $\mathrm{CH}_{4}$ and a mixture of $\mathrm{Ni}-\mathrm{Y}$ powders into the arc-jet plasma flame generated by a non-transferred plasma torch. Material evaluations of the synthesized product by transmission electron microscopy (TEM) and scanning electron microscopy (SEM) reveals that the growth rate of carbon nanotubes is very high, and that the produced carbon nanotubes mainly have a multi-walled structure even though the single-walled and coiled structures are rarely found. Since this process is continuously operable and easily scalable, it is expected to be a promising technique for large-scale commercial production of carbon nanotubes.
\end{abstract}

\section{*Keywords}

carbon nanotubes, arc-jet plasma, thermal plasma process, methane decomposition 


\section{Introduction}

Because of their unique properties, carbon nanotubes (CNTs) have a great potential of practical applications to field emission display [1], reinforcing component [2], hydrogen storage medium $[3,4]$, and so on. However, in order to realize their industrial applications, a continuous process for large-scale production of CNTs is essentially required. To date, several methods such as arc discharge [5,6], laser ablation [7], chemical vapor deposition (CVD) [8] and catalytic pyrolysis $[9,10]$ have widely used for CNTs production.

It is known that the conventional arc discharge method can generate highly crystallized CNTs by high temperature ambience (above $5000 \mathrm{~K}$ ) and achieve high production rates of soot in which the yield of CNT is not satisfactory. But, this approach has a drawback as an industrial technology due to its non-continuous process. The laser ablation can easily produce the CNT of high purity. However, their production rates are low, and the synthesis apparatus is expensive. The CVD method has merits to grow aligned CNT and control diameter or length of CNT. Unfortunately, this approach has difficulty in a continuous process because encapsulation of the metal particles, coated on a substrate by diffusion of carbon, stops the growth process. In the catalytic pyrolysis method, carbon nanotubes are produced in floating condition by introducing carbon and catalyst sources simultaneously into an electric furnace. Therefore, this method is recognized as a promising technique for large-scale production of industrial purpose due to its continuous process and easy scale-up.

As a modified catalytic pyrolysis method, we present here a promising technique for the continuous large-scale production of carbon nanotubes by decomposition of hydrocarbon in an arc-jet plasma. The arc-jet plasma is generated by blowing a cold 
plasma forming gas into the current path of dc arc discharge between two electrodes. Since the arc-jet plasma has thermal plasma properties of high temperature (5000-20000 K) and high velocity $(100-1000 \mathrm{~m} / \mathrm{s})$ [11], it has been widely used for plasma spray coating $[12,13]$, fine powder synthesis $[14,15]$, and hazardous waste treatment by pyrolysis of organic materials and melting of solid wastes [16]. When the arc-jet plasma technique is applied to the CNT synthesis, this approach is analogous to the conventional catalytic pyrolysis in respect that CNTs grow in a floating condition by injecting hydrocarbon gas and catalyst powder simultaneously into a hot discharge reactor zone. But, a thermal plasma reactor, instead of the electric furnace, is used as a heat source for decomposition of hydrocarbon and catalyst. Therefore, the de plasma torch used in the reactor can provide an optimum synthesis ambience of very high temperature, which is not achievable by the conventional electric furnace.

In the synthesis of CNTs by the arc-jet plasma method, its process features are expected as follows: i) It is possible to achieve the high rates of hydrocarbon decomposition and CNT growth resulted from the high temperature and enthalpy of thermal plasma; ii) This technique itself is regarded as a process for the metal nanoparticle synthesis [15] due to evaporation of the metal powder of large size in the thermal plasma of high temperature and enthalpy, and it does not use nano-sized metal powders or organic metals as a catalyst source in contrast with the conventional catalytic pyrolysis. The use of coarse powder is economically benefit for the catalyst supply; iii) This new process can be easily scale-up through a plasma power-up. Moreover, the plasma power-up can be achieved in a small volume without expansion of the hot plasma zone; iv) The synthesized materials can be easily collected because of 
one directional flow of the arc plasma jet, which permits a continuous synthesis process with a continuous supply of carbon and catalyst sources.

In this paper, we present a successful synthesis of carbon nanotubes by decomposition of methane $\left(\mathrm{CH}_{4}\right)$ by the arc-jet thermal plasma generated by a nontransferred plasma torch. The synthesized CNTs have been evaluated by the images of high-resolution transmission electron microscopy (TEM) and scanning electron microscopy (SEM). In our experimental study, we have found that the growth rate of CNTs in the arc-jet plasma is very high, and that mainly multi-walled CNTs are produced in high purity.

\section{Experimental}

A schematic diagram of the CNT synthesis system by hydrocarbon decomposition is shown in Fig. 1. The arc-jet plasma is generated by a non-transferred plasma torch, which consists of a tungsten cathode of conical shape and a copper anode of nozzle shape. While a plasma gas is introduced into a channel between the cathode and the nozzle anode, current paths form between the electrodes by dc arc discharge, and the resultant thermal plasma jet is ejected from the anode nozzle exit. Both electrodes are cooled by water in order to prevent them from erosion caused by enormous heat flux from the arc.

In this experiment, a cathode diameter of $12 \mathrm{~mm}$ and a conical angle of $60^{\circ}$, and an inner anode diameter of $6 \mathrm{~mm}$ were taken. A mixture gas of argon and helium was used as a plasma gas. Argon and helium flow rates were 10 slpm and 20 slpm, respectively. Methane was used as a hydrocarbon, nickel and yttrium powders (Sigma-Aldrich) were used as a metal precursor. $\mathrm{CH}_{4}$ of $1-4$ slpm and a metal powder mixture of $0.2 \mathrm{~g} / \mathrm{min}$ 
(4:1 atomic ratio) were introduced, through a stainless tube of $1.5 \mathrm{~mm}$ in inner diameter, into the arc-jet plasma near the anode nozzle exit. The mixture of nickel and yttrium powders has been commonly used by other researchers for the conventional arc discharge method $[17,18]$. The arc-jet plasma was generated with a dc current of $300 \mathrm{~A}$, and a loaded voltage of $48 \mathrm{~V}$. The reactor pressure was maintained at 500 Torr.

The CNT samples produced in this experiment were collected by a stainless steel collection tube for their material evaluation. They have been examined using highresolution TEM (JEOL JEM-3011), TEM (Philips CM-20), and field emission SEM (Hitachi S-4700), which were operated at 300, 200, and $10 \mathrm{kV}$, respectively.

\section{Results and Discussion}

After the synthesis processes, some soot was obtained from the collection tubes, and carbon nanotubes were found in the soot. All material analyses of the produced soot were conducted without going through a purification process. Figure 2 shows the macroscopic TEM images of the synthesized products of our experiment. As seen in a very low-magnification TEM image of Fig. 2(a), most of the produced materials are carbon nanotubes and a few amorphous carbons exist. The length of the CNTs is mainly observed to be several $\mu \mathrm{m}$, but long CNTs in a few tens $\mu \mathrm{m}$ are also found. It was observed in our synthesis experiment that carbon vapors are condensed within $20 \mathrm{~cm}$ of the plasma jet, of which velocity is generally known as higher than $100 \mathrm{~m} / \mathrm{s}[11,15]$. So, the growth rate of the carbon nanotubes in the jet plasma is estimated in a range of 3-30 $\mathrm{mm} / \mathrm{min}$. The growth rate reported in a CVD method was suggested as the order of $\mu \mathrm{m} / \mathrm{min}$ [19]. The diffusion flame synthesis method reported a higher growth rate of tens $\mu \mathrm{m} / \mathrm{min}$ [20]. Therefore, the growth rate of CNTs produced by our arc-jet thermal 
plasma method is much higher than that by other synthesis methods. An experimental study on the CVD method has reported that the growth rate increases with temperature [21]. Accordingly, it is inferred from this report that the much higher growth rates obtained in our experiment is due to the very high temperature of the arc-jet thermal plasma.

As observed in Fig. 2(b), outer diameters of the CNTs are widely distributed in a range of $10 \mathrm{~nm}-50 \mathrm{~nm}$ and inner diameters are narrowly distributed around $10 \mathrm{~nm}$. It was suggested that outer diameter of CNT depends mainly on the catalyst size evaporated during the synthesis process [22]. In the thermal plasma jet method, the initially injected metal particles of large size are first evaporated in the hot region of arc-jet thermal plasma and then condensed in the less hot region into nanoparticles, which act as catalysts for forming CNTs. In a metal nanoparticle synthesis experiment using an arc-jet plasma, metal particles of various sizes were synthesized by different temperature distributions [15]. Therefore, the wide distribution of outer diameters of CNTs obtained in our experiment appears to be caused by this general feature of arc-jet plasma synthesis. By taking advantage of this feature of thermal plasma synthesis, it is expected that the distribution of nanotubes diameters is controllable by regulating operation conditions of the plasma torch for getting a desired temperature distribution.

In Fig. 3, the carbon nanotubes of multi-walled $(a, b, c)$ and single-walled (c) structures are observed in the soot produced by our jet plasma method. Figure 2(b) also shows that most of produced carbon nanotubes are multi-walled ones. Assembles of single-walled CNTs as seen in Fig. 3(c) are rarely found in the soot. The single-walled CNTs layers appear to have large curvatures and relatively low crystallization degrees. 
In contrast, the multi-walled CNT layers in Fig. 4(a), (b) show smaller curvature and better crystallization degree compared to the single-walled CNTs.

Two different types of multi-walled CNTs are also observed in the TEM images in the Fig. 3 : (a) bamboo-like nanotubes with some internal caps and (b) cylindrical carbon nanotubes without internal caps. The appearance of the cylindrical CNTs obtained by the thermal plasma method is similar to that by the conventional catalytic pyrolysis method [23]. But the morphology of the bamboo-like nanotubes in our experiment is different from that in the conventional catalytic pyrolysis or CVD method [24,25]. Our CNTs have thick walls and small inner diameters, while those synthesized by the pyrolysis or CVD have very thin walls and large diameters of 50-150 nm, while ours have small diameters below $50 \mathrm{~nm}$.

Coiled carbon nanotubes as a peculiar structure are rarely found in the soot as seen in Fig. 3(d). as they have been sometimes produced by the CVD method [26,27]. The coiled carbon nanotubes are believed to have exceptional properties, though their applications have not been planed in an industrial scale yet [26]. The coiled carbon nanotubes can be described by their coil diameter and pitch. In our experiment, the coil diameter is about $100 \mathrm{~nm}$ and the pitch is about $50 \mathrm{~nm}$. In the CVD method, coil diameters are generally distributed in the range of 0.5-5 $\mu \mathrm{m}$ [28], and are much larger in comparison with our method.

Figure 4 shows end tips of the produced CNTs.: (a) not encapsulated with catalytic particle and (b) encapsulated with catalytic particle partly filled in their body. Encapsulated or filled CNTs indicate that the injected large sized metal powders have been sufficiently evaporated in the arc-jet thermal plasma of high temperature, and are acted as a catalyst for the carbon nanotube growth. 
Figure 5 shows the SEM image of the soot produced in our method reconfirms that carbon nanotubes are synthesized in high purity as observed previously from the TEM images of Fig. 2. This improved purity results of the arc-jet thermal plasma method mainly comes from its unique property of very high temperature (above $5000 \mathrm{~K}$ ), which never be achievable by the conventional catalytic pyrolysis method.

The production rate of carbon soot yet seems low in our laboratory-scale experiment. If $\mathrm{CH}_{4}$ of 1 slpm is fully extracted into solid carbon materials, the production rate becomes about $0.54 \mathrm{~g} / \mathrm{min}$. Because the processing conditions of our system are not fully optimized, the conversion rate of $\mathrm{CH}_{4}$ into carbon soot is below $10 \%$. But, our other study on a carbon black synthesis by this thermal plasma has shown that the conversion rate of hydrocarbon into the soot could be increased by using the optimization of processing conditions. In addition, even large amount of hydrocarbons can be easily decomposed by a plasma power-up, and structures of produced carbon nanotubes can be controlled by operation parameters.

\section{Conclusion}

We have demonstrated the synthesis method of carbon nanotubes by decomposition of methane in the arc-jet thermal plasma. The SEM and TEM images revealed that carbon nanotubes are successfully produced by this method. Especially, it was found that the growth rate of carbon nanotubes is very high, and mainly multiwalled CNTs in high purity and a few other structures of nanotubes were produced. However, the conversion rate of $\mathrm{CH}_{4}$ into carbon soot and the degree of crystallization of carbon nanotubes was somewhat low. 
The thermal plasma process is not yet optimized and further studies on production of carbon nanotubes by this method are required. But, we feel that this method is a promising technique for the large-scale commercial production of carbon nanotubes because of its numerous advantages of continuous operation, high growth rate of carbon nanotubes, easy scale-up of plasma power, and easy collection of products.

\section{Acknowledgment}

This work was supported by Korea Institute of Science and Technology Evaluation and Planning (KISTEP) of the Ministry of Science and Technology in Korea. 


\section{References}

[1] Q.H. Wang, A.A. Setlur, J.M. Lauerhaas, J.Y. Dai, E.W. Seelig and R.P.H. Chang, Appl. Phys. Lett. 72 (1998) 2912

[2] D. Qian, E.C. Dickey, R. Andrews and T. Rantell, Appl. Phys. Lett. 76 (2000) 2868

[3] C. Liu, Y.Y. Fan, M. Liu, H.T. Cong, H.M. Cheng and M.S. Dresselhaus, Science $286(1999) 1127$

[4] Anyuan Cao, Hongwei Zhu, Xianfeng Zhang, Xuesong Li, Dianbo Ruan, Cailu Xu, Binqing Wei, Ji Liang and Dehai Wu, Chem. Phys. Lett. 342 (2001) 510

[5] T.W. Ebbensen and P.M. Ajayan, Nature 358 (1992) 220

[6] S. Iijima and T. Ichihashi, Nature 363 (1993) 603

[7] Andreas Thess, Roland Lee, Pavel Nikolaev, Hongjie Dai, Pierre Petit, Jerome Robert, Chunhui Xu, Young Hee Lee, Seong Gon Kim, Andrew G. Rinzler, Daniel T. Colbert, Gustavo E. Scuseria, David Tomanek, John E. Fischer and Richard E. Smalley, Science 273 (1996) 483

[8] Young Chul Choi, Young Min Shin, Seong Chu Lim, Dong Jae Bae, Young Hee Lee, Byung Soo Lee, Dong-Chul Chung, J. Appl. Phys. 88 (2000) 4898

[9] H.M. Cheng, F.Li, L.L. He, X. Sun and M.S. Dresselhaus, Appl. Phys. Lett. 72 (1998) 3282

[10] Lijie Ci, Jinquan Wei, Bingqing Wei, Ji Liang, Cailu Xu and Dehai Wu, Carbon 39 (2001) 329

[11] Maher I. Boulos, IEEE T. Plasma Sci. 19 (1991) 1078

[12] Pierre Fauchais and Armelle Vardelle, IEEE T. Plasma Sci. 25 (1997) 1258

[13] M. Vardelle, A. Vardelle, A.C. Leger, P. Fauchais, and D. Gobin, J. Therm. Spray Tech. 4 (1995) 50 
[14] P. Fauchais, A. Vardelle and A. Denoirjean, Surf. Coat. Tech. 97 (1997) 66

[15] N. Rao, S. Girshick, J. Heberlein, P. McMurry, S. Jones, D. Hansen and B. Micheel, Plasma Chem. Plasma Process. 15 (1995) 581

[16] H.R. Snyder, C.B. Fleddermann and J.M. Gahl, Waste Manage. 16 (1996) 289

[17] Yoshinori Ando, Xiuluo Zhao, Kaori Hirahara, Kazutomo Suenaga, Shunji Bandow and Sumio Iijima, Chem. Phys. Lett. 323 (2000) 580

[18] Zujin Shi, Yongfu Lian, Fu Hui Liao, Xihuang Zhou, Zhennan Gu, Y. Zhang, S. Iijima, Hongdong Li, Kwok To Yue and Shu-Lin Zhang, J. Phys. Chem. Solids 61 (2000) 1031

[19] Z.F. Ren, Z.P. Huang, J.W.Xu, J.H. Wang, P. Bush, M.P. Siegal, P.N. Provencion, Science $282(1998) 1105$

[20] Randall L. Vander Wal, Thomas M. Ticich and Valerie E. Curtis, Chem. Phys. Lett. $323(2000) 217$

[21] Cheol Jin Lee, Jeunghee Park, Yoon Huh and Jeong Yong Lee, Chem. Phys. Lett. $343(2001) 33$

[22] W.Z. Li, D.Z. Wang, S.X. Yang, J.G. Wen and Z.F. Ren, Chem. Phys. Lett. 335 (2001) 141

[23] Yun Tack Lee, Nam Seo Kim, Jeunghee Park, Hae Beom Han, Young Sang Choi, Hyun Ryu and hwack Joo Lee, Chem. Phys. Lett. 372 (2003) 853

[24] Xianbao Wang, Wenping Hu, Yunqu Liu, chenfeng Long, Yu Xu, Shuqin Zhou, Daoben Zhu and Liming Dai, Carbon 39 (2001) 1533

[25] Cheol Jin Lee and Jeunghee Park, Carbon 39 (2001) 1891

[26] Seiji Motojima and Quiqin Chen, J. Appl. Phys. 85 (1999) 3919

[27] J.B. Bai, Mater. Lett. 57 (2003) 2629 
[28] Federic Fabry, Gilles Flamant and Laurent Fulcheri, Chem. Eng. Sci. 56 (2001) 2123 


\section{Figure Captions}

Fig. 1

Schematic diagram of an arc-jet thermal plasma reactor for carbon nanotubes synthesis by decomposition of methane

Fig. 2

Low-magnification TEM images of the morphology of as-grown carbon nanotubes show (a) length distributions of the CNTs and (b) diameter distributions of the synthesized CNTs

Fig. 3

TEM images show the carbon nanotubes of different structures produced by the arc-jet thermal plasma method: (a) bamboo-like multi-walled CNT (b) multi-walled CNT (c) single-walled CNTs, and (d) coiled CNTs

Fig. 4

High-resolution TEM images of as-grown carbon nanotubes: (a) open-tip CNTs (b) encapsulated tip and partially filled CNTs (c) wall structure of a cylindrical multiwalled CNT, and (d) wall structure of a bamboo-like multi-walled CNT

Fig. 5

SEM image of the carbon nanotubes obtained from the collection tubes 


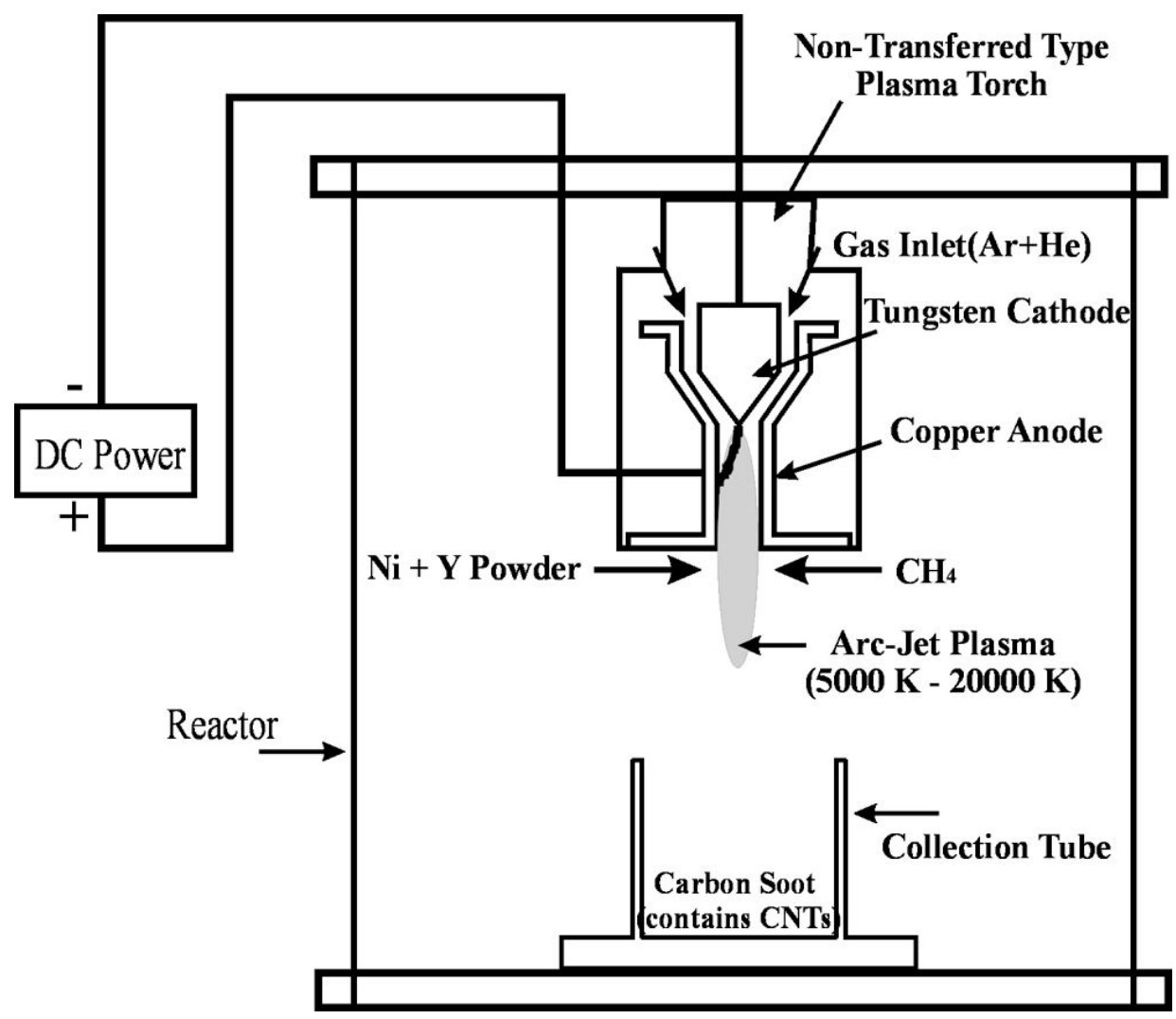

Fig. 1 


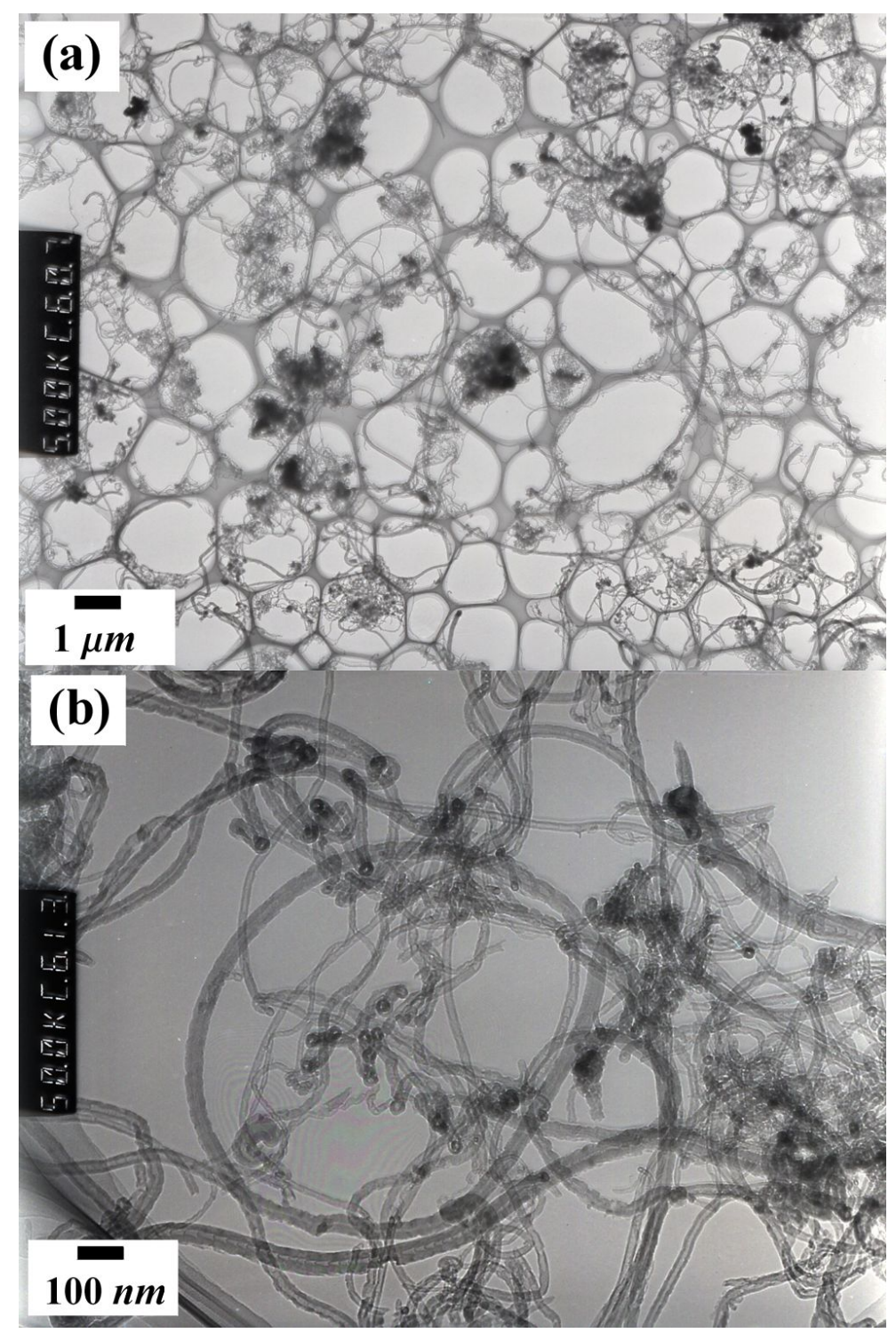

Fig. 2 


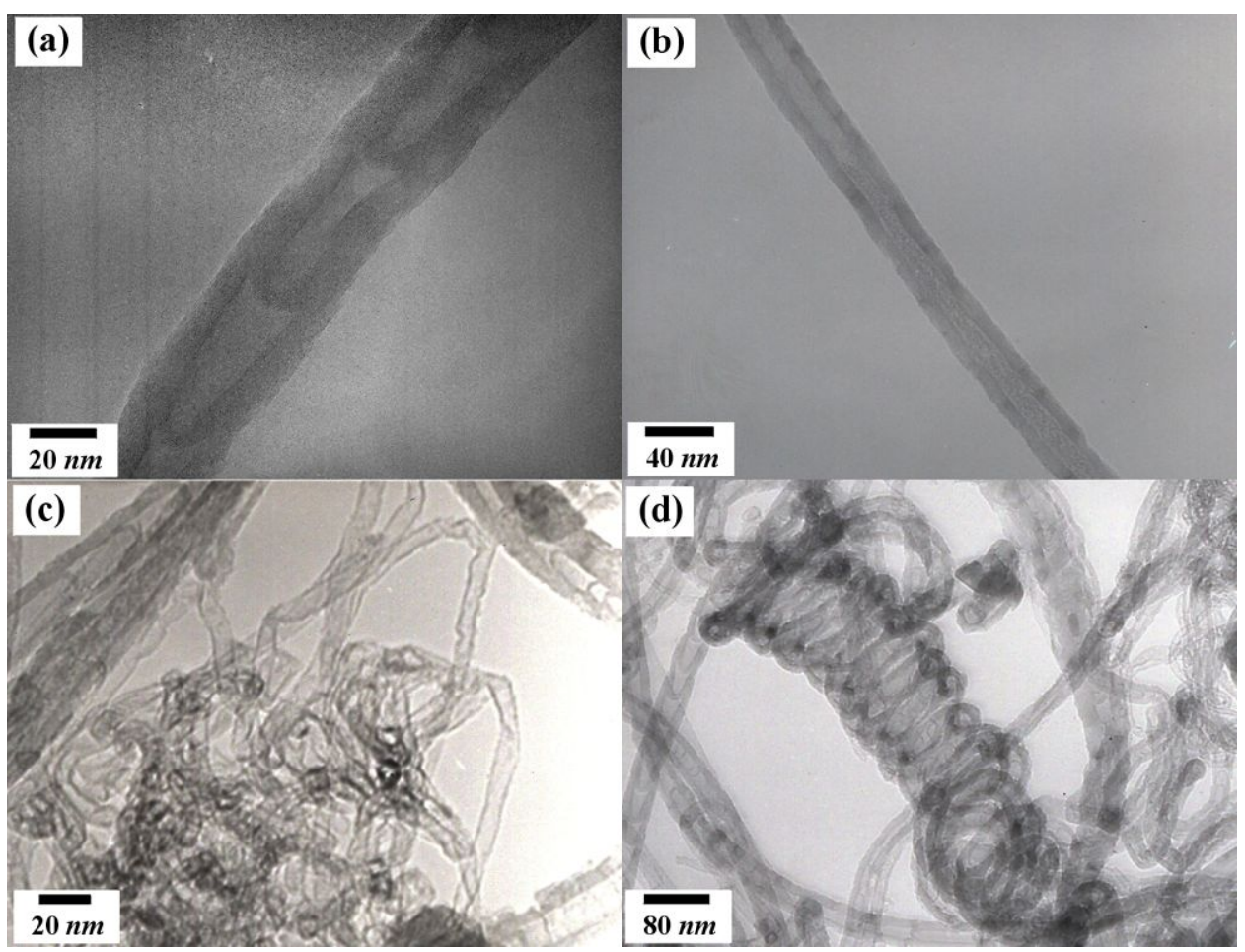

Fig. 3 


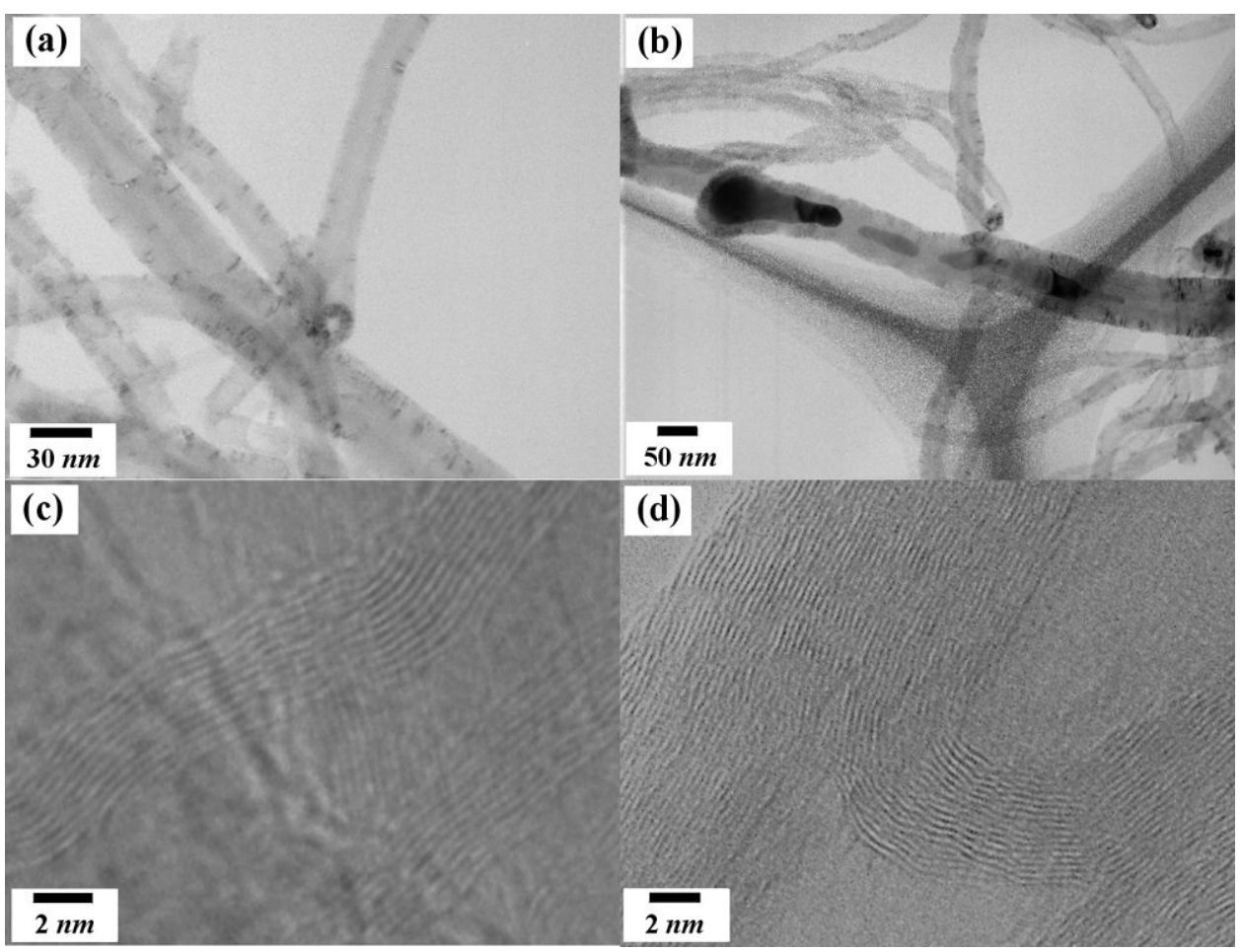

Fig. 4 


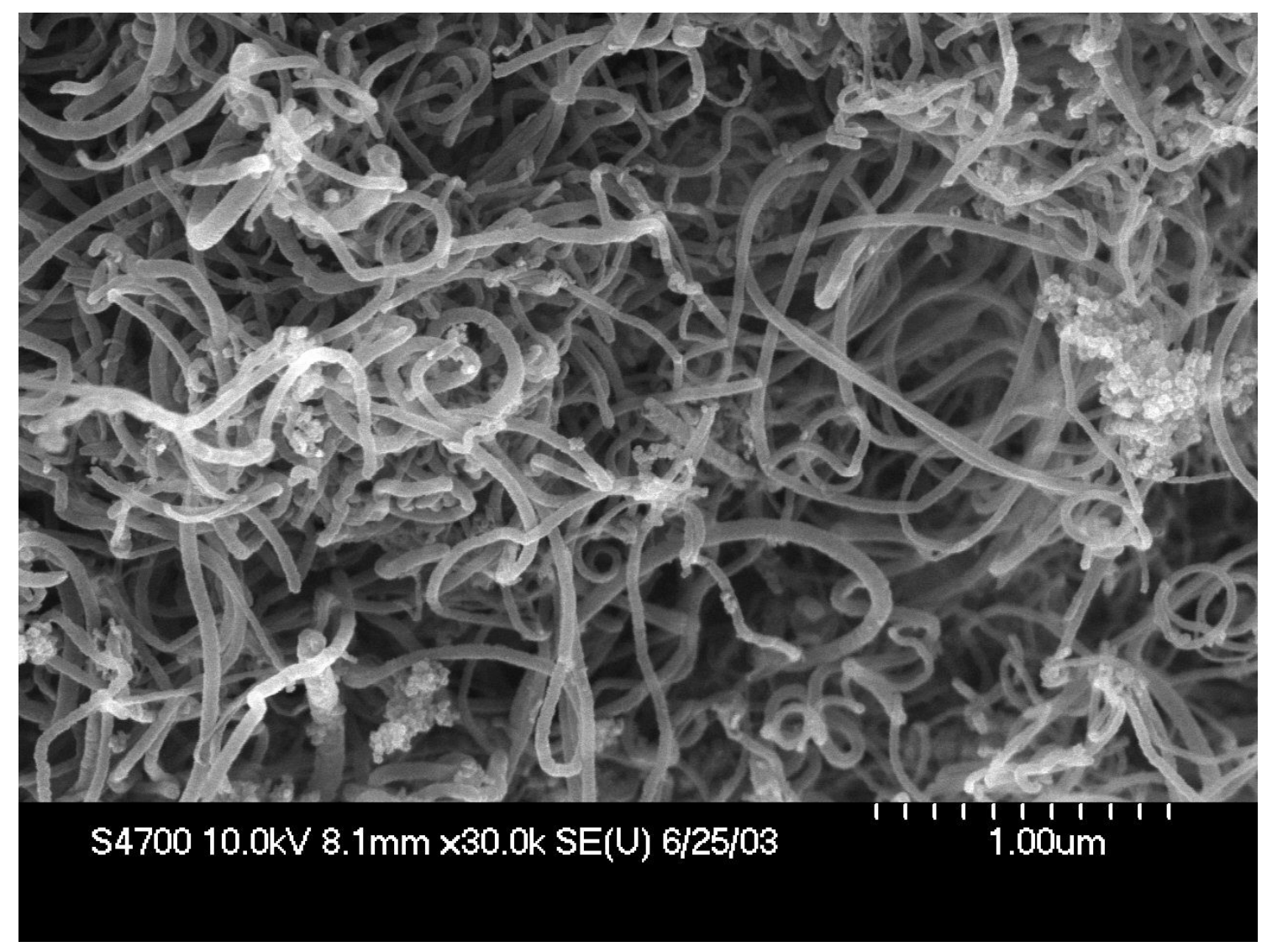

Fig. 5 